ARTICLE HISTORY: June: 17, 2021 Accepted: August 13, 2021 Published: August 19, 2021

ОПРЕДЕЛЕНИЕ СВОЙСТВ ЭЛЕМЕНТАРНЫХ ЧАСТИЦ

Якубовский Евгений Георгиевич,

пенсионер

\title{
DETERMINATION OF THE PROPERTIES OF ELEMENTARY PARTICLES
}

\author{
Yakubovsky Evgeny Georgievich \\ pensioner
}

\begin{abstract}
Аннотация. Частицы вакуума позволяют определять свойства элементарных частиц, но предстоит еще большая работа по развитию свойств частиц вакуума. Развитие квантовой электродинамики и стандартной модели насчитывает 100 лет. Между тем описание более низкого уровня масс и энергий насчитывает 10 лет, и то усилиями одного человека. При вычислении массы элементарных частиц и их магнитного момента используются линейные формулы, что снижает точность вычисления. Надо думать, о нелинейных поправках.

Abstract. Particles of vacuum make it possible to determine the properties of elementary particles, but there is still a lot of work to develop the properties of particles of vacuum. The development of quantum electrodynamics and the standard model goes back 100 years. Meanwhile, the description of a lower level of masses and energies is 10 years old, and that is through the efforts of one person. When calculating the mass of elementary particles and their magnetic moment, linear formulas are used, which reduces the accuracy of the calculation. One must think about nonlinear corrections.

Ключевые слова: свойства элементарных частиц, магнитный момент элементарных частиц, частицы вакуума
\end{abstract}

Key words: properties of elementary particles, magnetic moment of elementary particles, vacuum particles

Исследуется проблема описания свойств элементарных частиц с помощью частиц вакуума. Стандартная модель и квантовая электродинамика не могут решить эту проблему, так как нет перехода на более низкий уровень энергии и массы. Основу элементарных частиц составляют частицы вакуума, их описанием и надо заниматься. Если квантовая электродинамика и стандартная модель для каждой элементарной частицы выводит свою формулу со своими коэффициентами, то частицы вакуума в идеале определят одну формулу для счетного количества элементарных частиц. Дело в том, что формулы квантовой электродинамики и стандартной модели в своей электромагнитной части не содержат массу элементарной частицы и поэтому нет возможности отличить формулу одной частицы от другой. Кроме того, нет простого выражения для магнитного момента элементарной частицы.

Первый наскок на суммирование частиц вакуума с коэффициентом 1 не дал результата. Да и радиационные поправки вычислены с коэффициентом, отличным от 1. Но интегралов Фейнмана у частиц вакуума нет, нужна ему замена, она у меня есть, интеграл определяющий зависимость от времени волновой функции при одновременном вычислении координат частиц, с учетом решения уравнения Навье-Стокса. Но решение уравнения Навье-Стокса получено с точностью $10 \%$ из-за учета степени шероховатости и турбулентное решение без учета шероховатости не существует. А турбулентное решение, это связанные состояния, в которых надо учитывать степень шероховатости, а это облако электронов со своей шероховатостью. Пока нет точной формулы для магнитного момента элементарных частиц, займемся его аппроксимацией. По отклонению магнитного момента вычислены линейные коэффициенты, описывающие магнитные свойства четырех элементов электрона, мюона, нейтрона, протона. Формула определяет заложенные в нее экспериментальные значения отклонения магнитного момента. Отмечу, что в квантовой электродинамике и стандартной модели имеются отдельные формулы для каждой элементарной частицы, каждая со своими коэффициентами. Построена одна формула для 4 элементарных частиц.

Надо попробовать формулу в которой используется магнитный заряд см. [1]

$$
\begin{aligned}
& \mu_{m}=\sum_{k=1}^{\infty} \frac{e \hbar\left[-i m d_{k}\left(137 m_{p}^{0.5} / 4 m_{e}^{0.5}\right)^{4} m_{P l}\right]^{\frac{k}{-1}-\frac{1}{4}}}{2 m c} ; \\
& a_{m}=\sum_{k=2}^{\infty}\left[-i m d_{k}\left(137 m_{p}^{0.5} / 4 m_{e}^{0.5}\right)^{4} m_{P l}\right]^{\frac{k}{4}-\frac{1}{4}} \\
& \mu_{m}=\frac{e \hbar}{2 m c}\left(1+a_{m}\right)
\end{aligned}
$$


К сожалению данная формула дает не совпадающие с экспериментом значения. Необходимо вводить коэффициенты у каждого члена формулы. Тогда получится единая формула, описывающая все элементарные частицы. Кроме того, массы протона и нейтрона близка, а магнитный момент сильно отличается. Сведем задачу к линейному уравнению относительно четырех отклонений магнитного момента - электрона, мюона, нейтрона и протона. Система линейных уравнений имеет вид

$$
\begin{aligned}
& a_{n}=\sum_{k=0}^{3}\left[-i m_{n} d_{k}\left(137 m_{p}^{0.5} / 4 m_{e}^{0.5}\right)^{4} m_{P l}\right]^{\frac{k+2}{4}-\frac{1}{4}} x_{k}, n=0,1,2,3 \\
& d_{k}=6 \sqrt{2} \pi\left[\frac{k+2}{2(2 k+3)(2 k+5)}\right]^{\frac{2}{2 k+3}}
\end{aligned}
$$

Решение этой системы линейных уравнений представлено в виде таблицы

$$
\mathrm{x}=\left(\begin{array}{c}
9742.619933685 \\
-1051199.325865279 \\
24085930.696818016 \\
-146286874.69125909
\end{array}\right)
$$

Была проведена проверка, вычислен магнитный момент электрона, мюона, нейтрона и протона. Причем надо использовать значения масс элементарных частиц с высокой точностью, десяти десятичных единиц. Данные совпали. Это говорит о том, что матрица системы хорошо обусловлена. Лучше конечно определять коэффициенты $x_{k}, k=0,1,2,3$ из системы линейных уравнений, так как вычислены значения коэффициентов с ограниченной точностью, но точность коэффициентов достаточная. Причем возможно обобщение формулы на большое количество частиц при большом количестве неизвестных коэффициентов и, следовательно, описание большого количества элементарных частиц. Но возможно описание элементарных частиц по четверкам. Но данная формула не интерполяционная, в промежуточных значениях масс элементарных частиц данная формула врет.

\section{Выводы}

Существует перспективный метод определения магнитного момента элементарных частиц с использованием заряда магнитного поля с помощью частиц вакуума. Пока этот метод не разработан, а используется аппроксимация магнитного момента по экспериментальному материалу. Но эта аппроксимация дает вдохновляющие результаты. Удалось аппроксимировать экспериментальные значения магнитного момента для электрона, мюона, нейтрона и протона при использовании 4 членов аппроксимации. Значит при счетном количестве членов аппроксимации удастся получить одну формулу для магнитного момента счетного количества элементарных частиц. Но это идеал расчета, к которому нужно стремиться. Тогда подставляя в формулу для магнитного момента элементарных частиц точное значение массы, получим точное значение магнитного момента. А так как я стремлюсь получить значение массы элементарных частиц, пока с некоторой натяжкой с точностью $0.5 \%$, то возможно по квантовым числам определение магнитного момента элементарных частиц.

\section{Литература}

[1] Якубовский Е.Г. Магнитный заряд, которого так долго искали теоретики найден «Энциклопедический фонд России», 2021, 4 стр. http://russika.ru/userfiles/390_1622580058.pdf

\section{References}

[1] Yakubovsky E.G. Magnetic charge that theorists have been found so long found "Encyclopedic Fund of Russia", 2021, 4 pp. http://russika.ru/userfiles/390_1622580058.pdf 